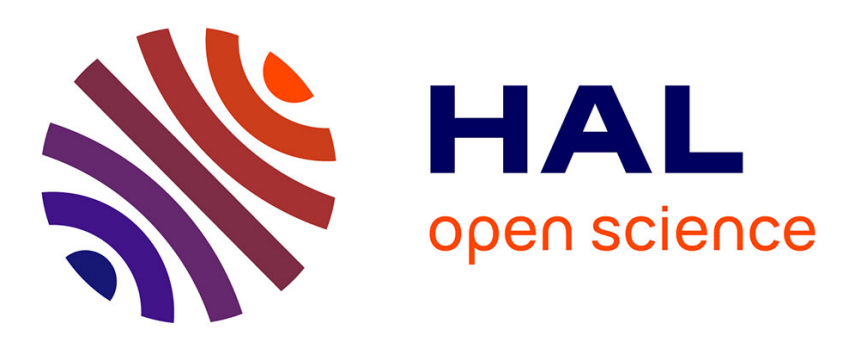

\title{
Evolutionary aspects of Anopheles-Plasmodium interactions
}

\author{
Louis A Lambrechts, Jacob C. Koella
}

\section{To cite this version:}

Louis A Lambrechts, Jacob C. Koella. Evolutionary aspects of Anopheles-Plasmodium interactions. Springer. Ecology of parasite-vector interactions, 3, Wageningen Academic Publishers, pp.123-137, 2013, Ecology and control of vector-borne diseases, 978-90-8686-744-8. 10.3920/978-90-8686-744-8_6 . pasteur-02010923

\section{HAL Id: pasteur-02010923 \\ https://hal-pasteur.archives-ouvertes.fr/pasteur-02010923}

Submitted on 8 Feb 2019

HAL is a multi-disciplinary open access archive for the deposit and dissemination of scientific research documents, whether they are published or not. The documents may come from teaching and research institutions in France or abroad, or from public or private research centers.
L'archive ouverte pluridisciplinaire HAL, est destinée au dépôt et à la diffusion de documents scientifiques de niveau recherche, publiés ou non, émanant des établissements d'enseignement et de recherche français ou étrangers, des laboratoires publics ou privés.

\section{(1) $\$(0)$}

Distributed under a Creative Commons Attribution - NonCommercial - ShareAlikel 4.0 
Ecology and Control of Vector-borne Diseases

Volume 3 - Ecology of vector-parasite interactions

Editors: C. J. M. Koenraadt \& W. Takken

Section 1 - Fundamental aspects of vector-parasite interactions

\title{
Chapter 2
}

\section{Evolutionary aspects of Anopheles-Plasmodium interactions}

\author{
Louis Lambrechts ${ }^{1} \&$ Jacob C. Koella ${ }^{2}$
}

${ }^{1}$ Insects and Infectious Diseases, CNRS URA 3012, Institut Pasteur, 25 rue du Docteur Roux, 75724 Paris Cedex 15, France

${ }^{2}$ Division of Biology, Imperial College London, Silwood Park Campus, Ascot, Berkshire SL5 7PY, United Kingdom 


\section{Abstract}

Interactions between Plasmodium parasites and their Anopheles vectors are central to the epidemiology of human malaria. This chapter highlights how an evolutionary perspective on Anopheles-Plasmodium interactions can provide important insights into the history, distribution and dynamics of malaria transmission. It focuses on three aspects: (1) the macroevolutionary history of malaria parasites in relation with their vectors, (2) the microevolutionary mechanisms shaping mosquito-parasite interactions and their impact on malaria transmission, and (3) the contribution of evolutionary concepts in the assessment of novel strategies to control malaria. The geographical distribution of diverse anopheline species and populations has played an important role in the past and present distribution of malaria. In particular, speciation processes and genetic differentiation of vectors may have been important drivers of the evolution of human malaria parasites. A full understanding of the epidemiology of malaria also requires careful consideration of the micro-evolutionary relationships between mosquitoes and malaria parasites. Malaria parasites have evolved, for example, to manipulate several parameters of the vector biology that are expected to increase their transmission. Finally, an evolutionary approach is useful for assessing the feasibility of innovative malaria control strategies such as the release of transgenic mosquitoes.

Considering the epidemiological feedback and the evolutionary response of wild mosquito and parasite populations is crucial for predicting the evolutionary trajectory of such a control measure. A major challenge for future research is to obtain quantitative, epidemiologically relevant estimates of the critical parameters underlying Anopheles-Plasmodium interactions in natural systems. 


\section{Introduction}

This chapter deals with evolutionary aspects of the interactions between malaria parasites and their mosquito vectors. Rather than exhaustively covering the topic, it describes examples of three aspects: macro-evolutionary patterns, the role of evolutionary ecology in shaping epidemiology, and the use of evolutionary ideas for control. The first section reviews approaches of population biology and phylogenetics that have shed light on the macroevolutionary history of malaria parasites in relation with their mosquito vectors. In particular, it describes the association of mammalian Plasmodium species with their specialization to Anopheles mosquito vectors, and the link between anopheline diversity and the current distribution of Plasmodium species and populations. The second section describes the microevolutionary mechanisms shaping interactions between Plasmodium and mosquitoes and their impact on malaria transmission. Special emphasis is put on recent insights provided by ecological immunology approaches into the role of mosquito immunity in malaria transmission. The third section focuses on the contribution of evolutionary concepts in the assessment of novel strategies to control malaria based on the release of transgenic mosquitoes. Specifically, it examines the conditions and consequences of the evolution of mosquito resistance to a lethal genetic construct (population suppression strategy) and evolution of parasite resistance to genetically engineered mosquito resistance (population replacement strategy). 


\section{Role of mosquitoes in the evolutionary history of Plasmodium parasites}

With the exception of a single species of lizard malaria, Plasmodium mexicanum, which is transmitted by sandflies, all known Plasmodium species use mosquitoes (Culicidae) as vectors (Ayala \& Lee 1970). While avian and reptilian malaria parasites are transmitted by many mosquito species from several genera (Levine 1988), all known Plasmodium species of mammals are transmitted by mosquitoes of the genus Anopheles. Recent phylogenetic analyses indicate that Plasmodium species infecting mammalian hosts form a well-supported clade associated with anopheline vectors (Fig. 1). Although this clade is paraphyletic (it includes parasites of the genus Hepatocystis), it suggests that expansion of Plasmodium parasites into mammals, including humans, corresponds with specialization into anophelines. This switch from culicine to anopheline mosquitoes, coincident with the expansion into mammals, is believed to have occurred once (Martinsen et al. 2008). It is unknown whether specialization into mosquitoes of the genus Anopheles is a cause or a consequence of Plasmodium expansion into mammals, for a phylogenetic pattern does not enable to infer the mechanism that drove evolution. Nevertheless, the striking associations between the phylogeny of Plasmodium parasites and their vertebrate and insect host taxa clearly reveal their three-way phylogenetic interdependence.

The evolutionary origin of P. falciparum, the deadliest human malaria parasite, has been a highly debated topic over the last two decades (Prugnolle et al. 2011). What has been controversial is whether P. falciparum became a human pathogen as a result of a transfer from ancestral birds, rodents or primates. The most recent evidence supports the origin of $P$. falciparum in gorillas (Liu et al. 2010). Although the exact time of the cross-species transmission event is unclear, this finding suggests a relatively recent origin of P. falciparum. 
It supports the view that P. falciparum emerged and expanded in the human population within the last 6,000 years (Rich et al. 1998; Volkman et al. 2001), coincidental with the emergence of agricultural societies in sub-Saharan Africa. The change in lifestyle from small nomadic groups to larger settled communities would have provided conditions for sustained $P$. falciparum transmission (Hume et al. 2003).

In addition, it has been proposed that the expansion of $P$. falciparum in the last few thousand years was facilitated by the speciation process of African mosquito vectors of the Anopheles gambiae species complex (Coluzzi et al. 2002). The most efficient $P$. falciparum vector species in the complex is An. gambiae sensu stricto (s.s.). Originally from the African rain forest, this highly anthropophilic species is thought be the product of a speciation process driven by environmental change following the agricultural revolution. Unlike most other anopheline species, An. gambiae s.s. mosquitoes typically breed in small, temporary, sunlit freshwater pools. Opening of the vegetation cover by human agriculture, therefore, would have created increased opportunities for larval breeding. Subsequent spread of An. gambiae s.s. from the rain forests into savanna areas probably occurred through close association with humans. Interestingly, this scenario is echoed by the current expansion of An. darlingi, the principal South American malaria vector, following deforestation of the Amazon Basin (Vittor et al. 2009).

In Africa, An. gambiae s.s. is further diversifying into sympatric ecotypes named $\mathrm{M}$ and $\mathrm{S}$ (Lawniczak et al. 2010). This diversification, probably promoted by adaptation to different larval habitats, may have important consequences for malaria transmission, for the adaptive divergence between incipient species includes that of immune genes conferring resistance to Plasmodium (White et al. 2010). Another example of diversification is a recently discovered 
outdoor-resting (exophilic) subgroup of An. gambiae s.s., which lives in sympatry with its indoor-resting (endophilic) counterparts (Riehle et al. 2011). This previously unknown population subgroup appears to be highly susceptible to wild P. falciparum isolates, and may play a key role in malaria transmission in places where disease control relies primarily on indoor-based vector control measures.

The geographical distribution of anopheline species and populations is clearly an important factor underlying the past and present distribution of human malaria (Hume et al. 2003). For example, the relatively inefficient malaria vectors in North Africa (An. pharoensis and An. sergentii) and in the Middle East (An. pulcherrimus) probably constrained the expansion of $P$. falciparum out of Africa (Hume et al. 2003). At a more local scale, the genetic structure of vector populations may also play an important role in shaping that of malaria parasites. Combined with the genetic structure of mosquito populations, genetic specificity of compatibility between malaria parasites and their mosquito vectors, both at the species (Billingsley \& Sinden 1997) and at the intraspecific level (Christophides et al. 2002; Lambrechts et al. 2005; Harris et al. 2010), promotes opportunities for parasite adaptation to local vector species and populations.

A textbook example of parasite local adaptation to vector species is provided by $P$. vivax, a human malaria species, in southern Mexico. Two malaria vector species are present at the southern tip of the region between the Pacific coast and the Guatemala border, but with largely non-overlapping geographic distributions. Anopheles albimanus is found in the costal region whereas An. pseudopunctipennis occurs in the foothills region. Using microsatellite markers to characterize parasite samples from 98 localities in an area of less than $100 \mathrm{~km}^{2}$, Joy et al. (2008) found that $P$. vivax consisted of three genetically distinct populations. One 
population (Fig. 2A; yellow dots) is distributed in the coastal ecoregion, where An. albimanus is found, whereas the two other populations (Fig. 2A; blue and red dots) are found in the foothills ecoregion, where An. pseudopunctipennis occurs. When they were artificially exposed to the three parasite populations in the laboratory, the two mosquito species were significantly more susceptible to the parasite genotypes encountered in their own ecoregion (Fig. 2B). The number of parasite oocysts developing on the mosquitoes' midgut was, on average, about three times higher in sympatric mosquito-parasite combinations than in allopatric pairs. This pattern is suggestive of parasite adaptation to local vectors, indicating that vector distribution can play an important role in generating parasite population structure (Joy et al. 2008). Although this process may also act at the mosquito population level within a single vector species, it has, to our knowledge, to date not been documented. In one study that examined the genetic co-structure between An. gambiae and P. falciparum populations, no pattern was found because of the lack of detectable genetic structure (Prugnolle et al. 2008). Because of the recent range expansion of vector and parasite populations, it is very challenging to find neutral markers that are genetically differentiated. In this case, reciprocal experimental infections provide an alternative test of local adaptation (Harris et al. 2012).

Understanding the factors shaping the adaptive evolution of Plasmodium populations has fundamental implications for public health (Mackinnon \& Marsh 2010). Arguably, too little attention has been paid to the role of vectors, relative to humans (e.g., Tanabe et al. 2010), in driving the evolution of malaria parasites at different spatial and temporal scales. In addition to the patterns of genetic compatibility between vector and parasites populations discussed above, variation in host preference among vectors may also have evolutionary and epidemiologically important consequences for malaria transmission (Lyimo \& Ferguson 2009). For example, evolutionary invasion analysis accounting for variation in vector 
anthropophily can help to predict the risk of emergence of $P$. knowlesi, usually considered a macaque malaria species, in the human population (Yakob et al. 2010). More generally, a deeper understanding of macro-evolutionary relationships between Plasmodium and their vectors, as well as with their vertebrate hosts, will provide important insights into the history, distribution and incidence of malaria. 


\section{Evolutionary ecology of mosquito-malaria interactions}

While the precedent section discussed macro-evolutionary patterns among species, this section considers evolutionary forces within single parasite-vector associations, thereby linking micro-evolutionary dynamics with epidemiology.

The epidemiology of malaria is generally summarized by a single metric, the basic reproductive number $\left(R_{0}\right)$, which represents the number of subsequent infections that arise from a single malaria case introduced into an entirely susceptible host population.

Mathematically, $R_{0}$ can be found from the Ross-Macdonald dynamics (Macdonald 1957) as

$$
R_{0}=\frac{m a^{2} e^{-\mu T}}{r \mu}
$$

where $m$ is the density of mosquitoes per human host, $a$ is the daily biting rate on humans, $\mu$ is the daily mortality rate of mosquitoes, $T$ is the duration in days of the parasite's developmental period within a mosquito (the extrinsic incubation period), and $r$ is the daily recovery rate of infected humans. More precisely, one must distinguish between the biting rate of uninfected mosquitoes on infectious humans, i.e. with gametocytes circulating in their blood $\left(a_{1}\right)$, and the biting rate of mosquitoes that are infectious, i.e. with sporozoites in their salivary glands $\left(a_{2}\right)$. One should also distinguish between the mortality rate of mosquitoes during the parasite's development $\left(\mu_{1}\right)$ and the mortality rate once the mosquitoes are infectious $\left(\mu_{2}\right)$. Malaria transmission also depends on successful completion of the parasite's development within the mosquito. This is summarized with a parameter, $b$, which combines the probability that the mosquito becomes infected upon biting a gametocytic human, the probability that the parasite survives through the extrinsic incubation period, and the 
probability that the bite of a mosquito with sporozoites in its salivary glands infects a human. Thus, equation (1) can be modified to

$$
R_{0}=\frac{m a_{1} a_{2} b e^{-\mu_{1} T}}{r \mu_{2}}
$$

It is striking to note, as Macdonald (1957) realized, that the intensity of malaria transmission is almost exclusively determined by traits related to either the mosquito or the mosquitoparasite interaction. Therefore, the evolutionary pressure for malaria parasites to increase the intensity of transmission will mostly be directed at their interaction with the vectors. Indeed, malaria parasites are able to manipulate most of the parameters in equation (2) in a way that is expected to increase $R_{0}$.

First, infection by the transmissible stages of malaria in humans increases their attractiveness to mosquitoes. Thus, in a semi-natural situation in western Kenya, about twice as many mosquitoes were attracted to children with gametocytes, the infectious stage of malaria in humans, than to children with no detectable infection or with a non-infectious stage of the parasite, but no gametocytes (Lacroix et al. 2005). In other words, the biting rate of uninfected mosquitoes on infectious humans (parameter $a_{l}$ in equation (2)) is higher than the biting rate on non-infectious humans, which is epidemiologically irrelevant.

Second, infection by the transmissible stages of malaria in mosquitoes increases their biting rate. In natural situations, mosquitoes with sporozoites, the infectious stage of malaria in the vector, are almost twice as likely to feed on more than one host than mosquitoes without sporozoites (Koella et al. 1998). One of the suspected underlying mechanisms is that sporozoites can reduce the activity of apyrase (Rossignol et al. 1984), an anticoagulant injected with the mosquito's saliva. As a consequence, mosquitoes take up less blood during the limited time available for a feeding attempt, which increases the probability that the 
mosquito bites a second time to top up its blood meal (Koella et al. 2002). Sporozoites also make mosquitoes more persistent if their blood-feeding attempts are not successful (Anderson et al. 1999). Overall, sporozoites increase the biting rate of infectious mosquitoes (parameter $a_{2}$ in equation (2)). Note that, as biting is risky (Day \& Edman 1984; Edman et al. 1984), sporozoites that manipulate the biting rate of mosquitoes also increase their feedingassociated mortality rate (Anderson et al. 2000). However, the selective pressure for increased biting overrides the pressure for lower mortality (Koella 1999).

Third, infection by the non-transmissible stages of malaria in mosquitoes decreases their biting rate. Malaria parasites at the oocyst stage do not profit from mosquito biting, because they cannot be transmitted. Rather, any (risky) biting reduces the probability that mosquitoes survive the parasite's extrinsic incubation period. Therefore, oocysts decrease the mosquitoes' biting rate by manipulating them in the opposite way of sporozoites: they decrease persistence if feeding is prevented (Anderson et al. 1999), and if feeding is interrupted they decrease the likelihood that the mosquitoes make a second feeding attempt to top up their blood meal (Koella et al. 2002). A recent study in Mozambique suggested that malaria parasites may also reduce their vector's biting rate by delaying gonotrophic cycles in An. funestus (Charlwood \& Tomas 2011).

Thus, four of the parameters in equation (2) are affected by manipulation of the mosquito's biting behavior by malaria parasites. Unfortunately, quantitative estimates for malariainduced changes of these parameters under field conditions are not available. Clearly, however, even small changes of each parameter in equation (2) will have a large effect on transmission intensity because they act on $R_{0}$ in a multiplicative way. Thus, if, say, each parameter is manipulated by the factor 2 , transmisssion is affected by the factor 8 . Accounting 
for manipulation of the biting behaviour of mosquitoes by malaria parasites may help to understand the unusually intense malaria transmission in endemic areas. Whereas, for example, the basic reproductive number (a measure of the intensity of transmission) of common childhood diseases ranges within about 10 to 30 (Anderson \& May 1991), it can reach more than 1000 for malaria (Smith et al. 2007). Importantly, ignoring the evolutionary forces that shape malaria-mosquito relationships would be misleading for entomological studies designed to understand the epidemiology of malaria. For example, mosquito biting rates on gametocytic humans, and biting rates and mortality rates of infected mosquitoes are epidemiologically relevant parameters, whereas biting and mortality rates of mosquitoes in the absence of infection are epidemiologically irrelevant. Measuring the latter would therefore not only underestimate the intensity of malaria transmission, potentially by a considerable amount, but might also underestimate the efforts needed for its control.

Evolutionary forces acting on the mosquito's biting rate also apply to parameter $b$ in equation (2), which represents the ability of malaria parasites to complete their development within the mosquito. As malaria parasites manipulate the mosquito's biting and mortality rates away from values that maximize their reproductive success (Koella 1999), natural selection is expected to favour mosquitoes that resist infection. In experimental infections, however, a substantial proportion of mosquitoes appear to be susceptible to malaria. For example, $P$. falciparum oocysts developed in $30 \%$ of An. gambiae mosquitoes exposed to gametocytes in a recent study in East Africa (Menge et al. 2006).

To date, most attempts to explain the observed variability of mosquito resistance to malaria have focused on elucidating its genetic basis. Selection procedures demonstrated the existence of a genetic basis for resistance (Collins et al. 1986; Vernick et al. 1995). Conclusions from 
these laboratory studies were confirmed with the identification of quantitative trait loci that explain the resistance of field-caught mosquitoes against malaria (Niaré et al. 2002; Menge et al. 2006; Riehle et al. 2006). In parallel, functional studies have revealed the complexity of molecular mechanisms underlying mosquito resistance to malaria. Although resistance also depends on other physiological factors than immunity (Vlachou et al. 2005), it is largely governed by a variety of immune responses, which are initiated by pattern recognition receptors regulating downstream effector mechanisms through signal modulation and transduction (for a recent review see Yassine \& Osta 2010). Despite considerable progress, it is debatable whether the current understanding, mostly based on molecular studies in laboratory models, of the immune response of anophelines against malaria adequately addresses the complexities of resistance and the intimate co-evolutionary processes between malaria and mosquitoes in nature (Boëte 2005; Cohuet et al. 2006; Michel et al. 2006; Boëte 2009; see related Chapter by Pike et al. in this book).

One complicating factor is that environmental variation can have a considerable effect on the expression of resistance, including its genetic basis. For example, symbiotic gut bacteria (Dong et al. 2009; Meister et al. 2009) and parasitic microsporidia (Bargielowski \& Koella 2009) strongly influence the prevalence of Plasmodium infection in mosquitoes. In addition, the concentration of sugar fed to mosquitoes after infection by malaria affects not only infection success, but also the differences among isofemale lines, i.e. the extent to which genes contribute to the observed variation of resistance (Lambrechts et al. 2006a; Hurd 2007).

More importantly, the interaction between malaria and mosquitoes is not governed by the mosquito's genes alone, but by the interaction between the mosquito's and the parasite's genes (Lambrechts et al. 2005; Harris et al. 2010), as is the case for many host-parasite 
interactions (Lambrechts et al. 2006b). Thus, a particular gene variant may make mosquitoes (partly) resistant against some malaria genotypes but not to others, while other gene variants make other mosquitoes resistant to other malaria genotypes. As a consequence, it is unlikely that a single resistance gene variant can confer resistance against all malaria genotypes, and that a single malaria genotype can overcome all mosquito resistance gene variants. Such a situation leads to either of two co-evolutionary outcomes: a stable coexistence of multiple resistance variants, or continuous cycling of the different variants (e.g., Leonard 1994). Due to such genotype-by-genotype interactions and their evolutionary dynamics, it is not very surprising that attempts to find adaptive signatures in anti-Plasmodium mosquito immune genes, which test for positive, directional selection on gene variants, were inconclusive so far (Slotman et al. 2007; Obbard et al. 2008; Parmakelis et al. 2008).

The realization that studies of laboratory systems provide limited insights into the intimately co-evolved systems of malaria and mosquitoes in nature is bringing molecular biologists and evolutionary ecologists together to study the variation of mosquito resistance against malaria in their natural ecological and evolutionary context, an emerging field referred to as the “ecological immunology” of mosquito-malaria interactions (Tripet et al. 2008; Tripet 2009). This approach focuses on questions of function and adaptation, with less emphasis on the molecular mechanisms of immunity and resistance (Schmid-Hempel 2005). At the centre of this approach is the idea that a balance between evolutionary costs and benefits determines the level of resistance in a population. On the one hand, as discussed above, malaria parasites manipulate the mosquito's biting rate and mortality away from the mosquito's optimum. Malaria infection can also directly affect the mosquito's physiology, reducing its fecundity and survival (reviewed in Tripet et al. 2008). Because of such fitness costs associated with malaria infection, resistance is clearly beneficial to the mosquito. On the other hand, the 
mosquito's immune response and resistance also involve costs, which are thought to result in part from physiological trade-offs due to shared pathways between immune responses and other physiological functions (Tripet et al. 2008). Such an evolutionary trade-off was demonstrated by the observation that mosquitoes selected for rapid development have a weaker melanisation immune response than those selected for slow development (Koella \& Boëte 2002). Because of the genetic correlation between the two traits, any evolutionary change in one trait would be associated with changes in the other trait. Overall, it is expected that evolution will increase resistance (resulting from increased immune function) only to the level where its benefit is balanced by its cost.

That evolution balances costs and benefits may be the main mechanism for the maintenance of susceptibility in natural populations (Boëte \& Koella 2003; Tripet et al. 2008; see related Chapter by Pike et al. in this book). This evolutionary perspective may also help to understand why the number of malaria oocysts observed in naturally infected mosquitoes is low. Indeed, most mosquitoes infected with $P$. falciparum in natural populations have a single oocyst of the parasite (e.g., Lyimo \& Koella 1992), whereas in laboratory systems they harbour many more. One possible explanation is that evolution has led to a balance between the disadvantages and advantages of resistance. On the one hand, weak resistance would lead to high oocyst loads, which would result in substantial damage. For example, the ability to manipulate biting rate increases with parasite load. Re-analysis of the data in Koella et al. (1998) shows that whereas about $22 \%$ of the mosquitoes infected with sporozoites bit more than one person if their sporozoite load was lower than the median, about $36 \%$ did so if their sporozoite load was greater than the median. On the other hand, a very effective immune response leading to complete parasite clearance is likely to be very costly, both in terms of expenditure of energy and in terms of the production of toxic compounds such as melanin. 
Therefore, an intermediate level of resistance, enabling low-level infection with moderate pathogenic effects, may be optimal for the mosquito. While some preliminary data support this idea (Boëte et al. 2004), the idea has not been formally tested.

This section emphasized that fully understanding the epidemiology of malaria and the biology of its vectors requires careful consideration of the micro-evolutionary relationships between mosquitoes and malaria parasites. Although the influence of evolutionary forces on the vectorial capacity of mosquitoes for malaria has been clearly demonstrated by proof-ofprinciple, qualitative experiments, much work remain to be done to turn predictions and speculations into quantitative assessments. Understanding how the co-evolution of malaria and mosquitoes affects the epidemiology of malaria and the pattern of resistance of mosquitoes in nature remains a major challenge. 


\section{Evolutionary assessment of genetically-modified mosquitoes for malaria}

\section{control}

This last section is intended to illustrate how the approach of evolutionary ecology described in the previous section can be used to assess the feasibility of a malaria control strategy. As it is beyond the scope of this chapter (and current knowledge) to give a detailed and quantitative prediction, we restrict ourselves to a few selected studies describing critical aspects rather than giving an extensive review of the relevant literature. In the prospect of releasing genetically-modified mosquitoes to control or eliminate malaria, it is crucial to understand the potential evolutionary trajectory and evaluate the impact on the incidence of disease. More details on this topic are available in several earlier publications (Boëte \& Koella 2002; Koella \& Boëte 2003; Koella \& Zaghloul 2008).

Failure of existing malaria control methods have stimulated research to develop novel, innovative strategies. In the last two decades, breakthroughs in the molecular genetics of mosquitoes have provided the groundwork for implementing vector control strategies based on the release of genetically-modified mosquitoes (Alphey et al. 2002; Christophides 2005; Catteruccia 2007). These strategies aim at either replacing the existing wild vector population with engineered vectors that are refractory to the pathogen (population replacement) (Nirmala \& James 2003) or eliminating the wild vector population using a genetic system that reduces reproductive capacity (population suppression) (Alphey et al. 2008). In the case of a population replacement strategy, the task is two-fold. It consists of (1) genetically engineering mosquitoes that are refractory to the pathogen and (2) driving the genetic construct conferring refractoriness to fixation into the wild target population. Although transgenic mosquito lines impaired in malaria transmission have been created in the laboratory (Ito et al. 2002; Corby- 
Harris et al. 2010), much remains to be done before these proofs-of-principle are converted into a feasible malaria control tool (Hill et al. 2005). One of the major challenges ahead is optimization of genetic drive systems to deliver the refractory transgenes into wild vector populations (Marshall \& Taylor 2009). Naturally occurring selfish genetic elements, such as transposons, meiotic drive genes, endosymbiotic bacteria or homing endonuclease genes, are promising candidates to develop such genetic drive systems (Sinkins \& Gould 2006). A synthetic selfish genetic element has been shown to drive population replacement in Drosophila (Chen et al. 2007), and very recently in An. gambiae (Windbichler et al. 2011).

By contrast, genetic strategies of vector population suppression generally do not require such a gene drive system because they rely on the repeated mass releases of transgenic sterile insects (Alphey et al. 2008). The disabled insects mate with wild individuals in the target population, thereby reducing its reproductive output, and potentially resulting in the subsequent collapse of the wild population if sufficient numbers of sexually competitive transgenic insects are released. The potential of this strategy has been recently supported by the successful elimination of caged populations of the dengue virus vector Aedes aegypti under semi-field conditions (Wise de Valdez et al. 2011), and during a recent open-field trial on the Cayman Islands (Harris et al. 2011). Thus, despite reduced mating competitiveness and smaller adult body size of genetically-modified mosquitoes compared to their wild type counterparts (Bargielowski et al. 2011a; Bargielowski et al. 2011b), frequent releases can compensate for the fitness costs.

There are at least three critical aspects that are often overlooked in the development of malaria control strategies based on genetically-modified mosquitoes. First, in the case of a population replacement strategy, the epidemiological feed back on the population genetic 
process influences the conditions allowing fixation of the trait of interest. The spread of an allele conferring refractoriness to malaria in a mosquito population was examined by a model combining population genetics with epidemiology (Boëte \& Koella 2002). This model demonstrated that because the change in malaria prevalence in the human population feeds backs onto the mosquito fitness, which depends in part on the evolutionary costs and benefits of refractoriness, fixation of refractoriness requires a genetic drive mechanism that increases the frequency of inheritance of the genetic element compared to regular Mendelian inheritance. Although refractoriness confers a clear evolutionary advantage to the mosquitoes when parasite prevalence is high (Marrelli et al. 2007), this advantage decreases progressively when transmission and parasite prevalence are decreasing due to the spread of refractoriness (Lambrechts et al. 2008). Luckily, a moderate efficacy of the drive mechanism is enough to spread a refractoriness allele to fixation in most situations (Boëte \& Koella 2002).

Second, the evolutionary response of the parasite can significantly threaten the effectiveness of the intervention outcome. Parasites are often able to evade or suppress the defence mechanisms of their hosts, including Plasmodium parasites in their mosquito vectors (Boëte et al. 2004; Lambrechts et al. 2007). A co-evolutionary model predicted that the optimal level of malaria parasite investment into evasion or suppression mechanisms depends on the relative investment into defence mechanisms of the mosquito (Koella \& Boëte 2003). Thus, in the case of a vector population replacement strategy, introducing a genetic construct conferring refractoriness to malaria infection may alter this co-evolutionary balance towards greater investment of the parasite into evading or suppressing the refractoriness mechanism. This would result in a decreased efficacy of refractoriness, which was showed to dramatically reduce the impact of the control program on malaria prevalence in the human population (Boëte \& Koella 2002; Koella \& Zaghloul 2008). One way of avoiding, or at least delaying, 
this co-evolutionary response may be to transform mosquitoes with mechanisms of refractoriness that are not found in natural systems. It would be less likely that standing genetic variation for suppressing or evading the refractory mechanism is already present in the parasite population. Therefore, the co-evolutionary response would be delayed until the necessary mutations have arisen.

Third, in the case of a population suppression strategy, it is important to carefully consider the evolutionary response of the wild vector population. For example, evolution of resistance to the lethal mechanism is a potential threat to population suppression strategies based on the release of mosquitoes carrying a dominant, repressible, lethal genetic construct. Mathematical modelling showed that although resistant alleles are unable to spread to fixation, they can become more common than the alternative susceptible allele and therefore have a detrimental impact on the release program (Alphey et al. 2011).

In conclusion, there is much to gain by including an evolutionary approach to assess the feasibility of using genetically-modified mosquitoes for malaria control. Not only evolutionary mathematical modelling, but also experimental evolutionary ecology, can provide important insights into the potential success of these strategies (Boëte \& Koella 2003). 


\section{Conclusions and Perspectives}

The evolutionary forces underlying the vectorial capacity of anopheline mosquitoes for malaria parasites are still poorly understood. One of the greatest challenges for future research is to obtain quantitative, epidemiologically relevant estimates of some of the critical parameters underlying malaria transmission. This will only be achieved by studying natural systems of Anopheles-Plasmodium interactions. At least in studies designed with the goal of understanding malaria epidemiology, efforts must be made so that experiments emulate more closely the natural conditions that are meaningful for parasite transmission. For example, whether malaria parasites kill their vectors has been largely debated (Ferguson \& Read 2002), but most of the studies on the effect of malaria infection on mosquito survival ignore feedingassociated mortality, which is likely to account for most of the mortality in nature. A related challenge is to account for the influence of manifold abiotic factors on mosquito-parasite interactions and their implications for malaria transmission. For example, the impact of shortterm temperature fluctuations on the development of malaria parasites in mosquitoes has recently been revealed, either speeding up or slowing down processes depending on the mean temperature (Paaijmans et al. 2010). This highlights the need for a better understanding of the mechanistic link between environmental heterogeneity and vectorial capacity. More generally, deciphering mosquito-parasite dynamics and co-evolution will require moving from laboratory conditions to more realistic systems. 


\section{Figures}

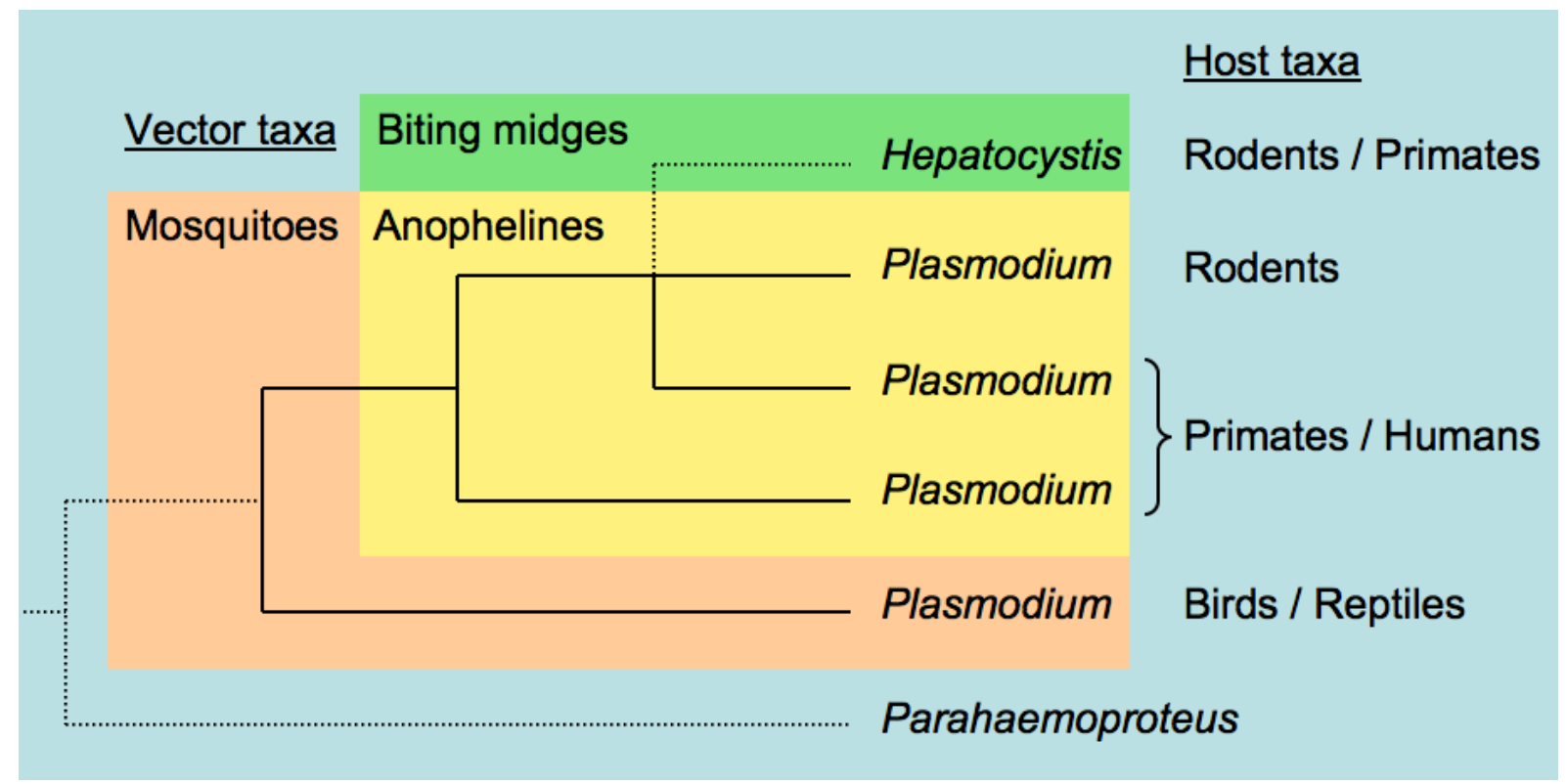

Figure 1. Schematic relationships between the phylogeny of Plasmodium parasites and their vertebrate host and vector taxa. All clades shown are well supported across different phylogenetic methods. This simple phylogram ignores the only Plasmodium species transmitted by sandflies. Adapted from Martinsen et al. (2008). 
A

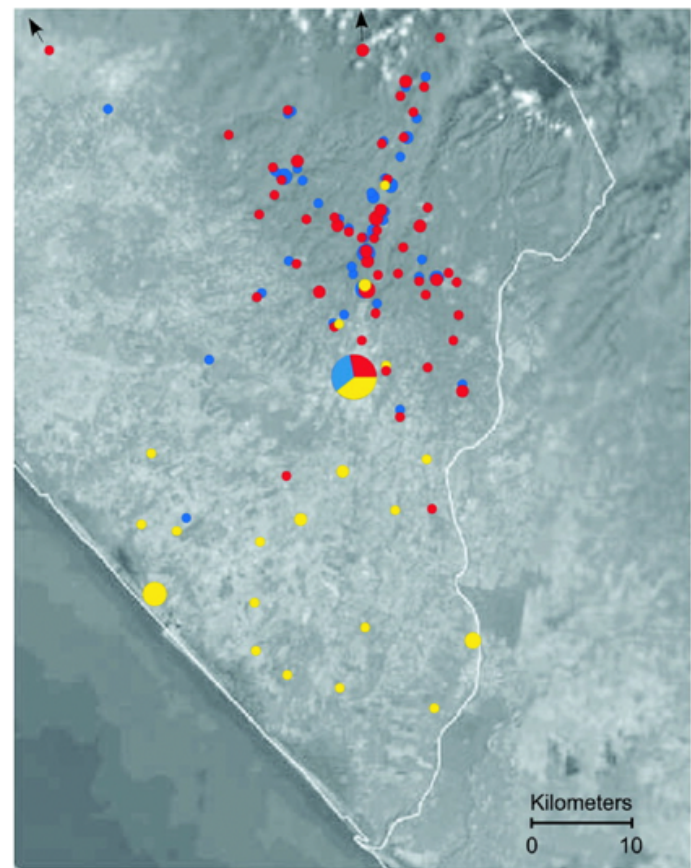

\section{B}

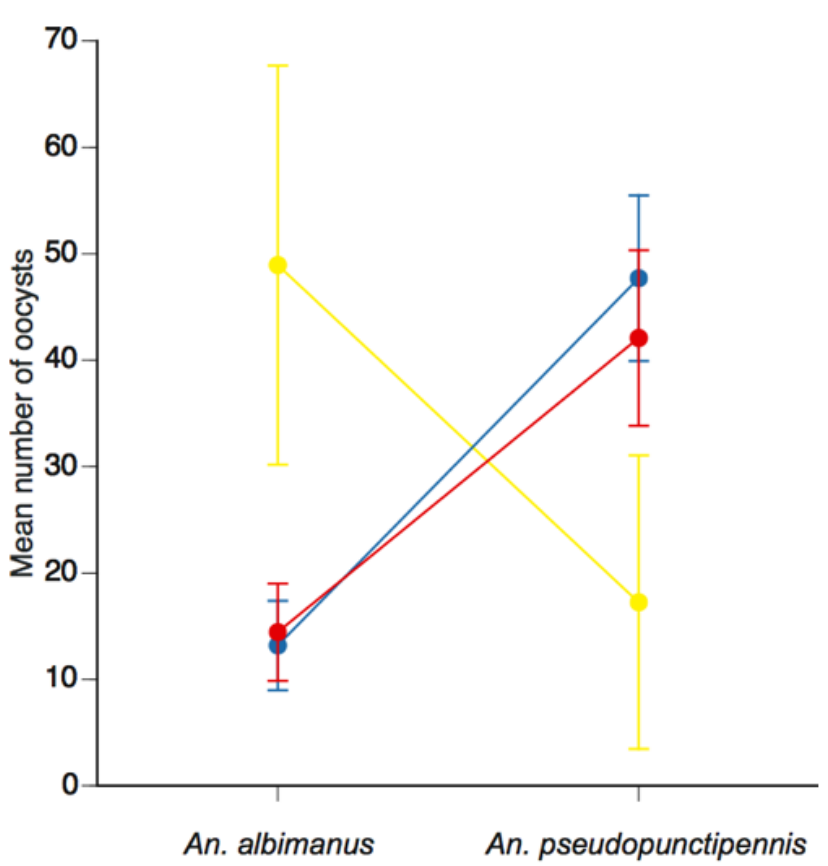

Figure 2. Pattern of adaptation to local vectors in Plasmodium vivax malaria. (A) Spatial genetic structure of $P$. vivax populations in the Pacific coastal region of southern Chiapas State, Mexico. Each sample was assigned to one of three identified genetically different populations, indicated by different colours. Circle size is proportional to the frequency of the sample, with the exception of the town of Tapachula in the centre. (B) Infection intensities observed in experimental infections of each combination of the three parasite populations and the two mosquito species. Lines represent the parasite genotypes according to the colours of panel A. Vertical bars are $95 \%$ confidence intervals. The interaction between mosquito species and parasite genotype is highly statistically significant $(P<0.0001)$. Modified, with permission, from Joy et al. (2008). 


\section{References}

Alphey L., Beard C.B., Billingsley P., Coetzee M., Crisanti A., Curtis C., Eggleston P., Godfray C., Hemingway J., Jacobs-Lorena M., James A.A., Kafatos F.C., Mukwaya L.G., Paton M., Powell J.R., Schneider W., Scott T.W., Sina B., Sinden R., Sinkins S., Spielman A., Toure Y. \& Collins F.H. (2002). Malaria control with genetically manipulated insect vectors. Science, 298, 119-21.

Alphey L., Nimmo D., O'Connell S. \& Alphey N. (2008). Insect population suppression using engineered insects. Adv Exp Med Biol, 627, 93-103.

Alphey N., Bonsall M.B. \& Alphey L. (2011). Modeling resistance to genetic control of insects. J Theor Biol, 270, 42-55.

Anderson R.A., Knols B.G. \& Koella J.C. (2000). Plasmodium falciparum sporozoites increase feeding-associated mortality of their mosquito hosts Anopheles gambiae s.l. Parasitology, 120, 329-33.

Anderson R.A., Koella J.C. \& Hurd H. (1999). The effect of Plasmodium yoelii nigeriensis infection on the feeding persistence of Anopheles stephensi Liston throughout the sporogonic cycle. Proc Biol Sci, 266, 1729-33.

Anderson R.M. \& May R.M. (1991). Infectious Diseases of Humans: Dynamics and Control. Oxford University Press, Oxford, UK.

Ayala S.C. \& Lee D. (1970). Saurian malaria: development of sporozoites in two species of phlebotomine sandflies. Science, 167, 891-2.

Bargielowski I., Alphey L. \& Koella J.C. (2011a). Cost of mating and insemination capacity of a genetically modified mosquito Aedes aegypti OX513A compared to its wild type counterpart. PLoS ONE, 6, e26086. 
Bargielowski I. \& Koella J.C. (2009). A possible mechanism for the suppression of Plasmodium berghei development in the mosquito Anopheles gambiae by the microsporidian Vavraia culicis. PLoS One, 4, e4676.

Bargielowski I., Nimmo D., Alphey L. \& Koella J.C. (2011b). Comparison of life history characteristics of the genetically modified OX513A line and a wild type strain of Aedes aegypti. PLoS ONE, 6, e20699.

Billingsley P.F. \& Sinden R.E. (1997). Determinants of Malaria-Mosquito Specificity. Parasitol Today, 13, 297-301.

Boëte C. (2009). Anopheles mosquitoes: not just flying malaria vectors... especially in the field. Trends Parasitol, 25, 53-5.

Boëte C. (2005). Malaria parasites in mosquitoes: laboratory models, evolutionary temptation and the real world. Trends Parasitol, 21, 445-447.

Boëte C. \& Koella J.C. (2002). A theoretical approach to predicting the success of genetic manipulation of malaria mosquitoes in malaria control. Malar J, 1, 3 .

Boëte C. \& Koella J.C. (2003). Evolutionary ideas about genetically manipulated mosquitoes and malaria control. Trends Parasitol, 19, 32-8.

Boëte C., Paul R.E. \& Koella J.C. (2004). Direct and indirect immunosuppression by a malaria parasite in its mosquito vector. Proc R Soc Lond B Biol Sci, 271, 1611-5.

Catteruccia F. (2007). Malaria vector control in the third millennium: progress and perspectives of molecular approaches. Pest Manag Sci, 63, 634-40.

Chen C.H., Huang H., Ward C.M., Su J.T., Schaeffer L.V., Guo M. \& Hay B.A. (2007). A synthetic maternal-effect selfish genetic element drives population replacement in Drosophila. Science, 316, 597-600.

Christophides G.K. (2005). Transgenic mosquitoes and malaria transmission. Cell Microbiol, $7,325-33$. 
Christophides G.K., Zdobnov E., Barillas-Mury C., Birney E., Blandin S., Blass C., Brey P.T., Collins F.H., Danielli A., Dimopoulos G., Hetru C., Hoa N.T., Hoffmann J.A., Kanzok S.M., Letunic I., Levashina E.A., Loukeris T.G., Lycett G., Meister S., Michel K., Moita L.F., Muller H.M., Osta M.A., Paskewitz S.M., Reichhart J.M., Rzhetsky A., Troxler L., Vernick K.D., Vlachou D., Volz J., von Mering C., Xu J., Zheng L., Bork P. \& Kafatos F.C. (2002). Immunity-related genes and gene families in Anopheles gambiae. Science, 298, 159-65.

Cohuet A., Osta M.A., Morlais I., Awono-Ambene P.H., Michel K., Simard F., Christophides G.K., Fontenille D. \& Kafatos F.C. (2006). Anopheles and Plasmodium: from laboratory models to natural systems in the field. EMBO Rep, 7, 1285-9.

Collins F.H., Sakai R.K., Vernick K.D., Paskewitz S., Seeley D.C., Miller L.H., Collins W.E., Campbell C.C. \& Gwadz R.W. (1986). Genetic selection of a Plasmodium-refractory strain of the malaria vector Anopheles gambiae. Science, 234, 607-10.

Coluzzi M., Sabatini A., della Torre A., Di Deco M.A. \& Petrarca V. (2002). A polytene chromosome analysis of the Anopheles gambiae species complex. Science, 298, 14158.

Corby-Harris V., Drexler A., Watkins de Jong L., Antonova Y., Pakpour N., Ziegler R., Ramberg F., Lewis E.E., Brown J.M., Luckhart S. \& Riehle M.A. (2010). Activation of Akt signaling reduces the prevalence and intensity of malaria parasite infection and lifespan in Anopheles stephensi mosquitoes. PLoS Pathog, 6, e1001003.

Day J.F. \& Edman J.D. (1984). Mosquito engorgement on normally defensive hosts depends on host activity patterns. J Med Entomol, 21, 732-40.

Dong Y., Manfredini F. \& Dimopoulos G. (2009). Implication of the mosquito midgut microbiota in the defense against malaria parasites. PLoS Pathog, 5, e1000423. 
Edman J.D., Day J.F. \& Walker E.D. (1984). Field confirmation of laboratory observations on the differential antimosquito behavior of herons. Condor, 86, 91-92.

Ferguson H.M. \& Read A.F. (2002). Why is the effect of malaria parasites on mosquito survival still unresolved? Trends Parasitol, 18, 256-61.

Harris C., Lambrechts L., Rousset F., Abate L., Nsango S.E., Fontenille D., Morlais I. \& Cohuet A. (2010). Polymorphisms in Anopheles gambiae Immune Genes Associated with Natural Resistance to Plasmodium falciparum. PLoS Pathog, 6.

Harris C., Morlais I., Churcher T.S., Awono-Ambene P., Gouagna L.C., Dabire R.K., Fontenille D. \& Cohuet A. (2012). Plasmodium falciparum produce lower infection intensities in local versus foreign Anopheles gambiae populations. PLoS ONE, 7, e30849.

Hill C.A., Kafatos F.C., Stansfield S.K. \& Collins F.H. (2005). Arthropod-borne diseases: vector control in the genomics era. Nat Rev Microbiol, 3, 262-8.

Hume J.C., Lyons E.J. \& Day K.P. (2003). Human migration, mosquitoes and the evolution of Plasmodium falciparum. Trends Parasitol, 19, 144-9.

Hurd H. (2007). Nature or nurture in mosquito resistance to malaria? Trends Parasitol, 23, $135-8$.

Ito J., Ghosh A., Moreira L.A., Wimmer E.A. \& Jacobs-Lorena M. (2002). Transgenic anopheline mosquitoes impaired in transmission of a malaria parasite. Nature, 417, $452-5$.

Joy D.A., Gonzalez-Ceron L., Carlton J.M., Gueye A., Fay M., McCutchan T.F. \& Su X.Z. (2008). Local adaptation and vector-mediated population structure in Plasmodium vivax malaria. Mol Biol Evol, 25, 1245-52.

Koella J.C. (1999). An evolutionary view of the interactions between anopheline mosquitoes and malaria parasites. Microbes Infect, 1, 303-8. 
Koella J.C. \& Boëte C. (2003). A model for the coevolution of immunity and immune evasion in vector-borne diseases with implications for the epidemiology of malaria. Am Nat, $161,698-707$.

Koella J.C. \& Boëte C. (2002). A genetic correlation between age at pupation and melanization immune response of the yellow fever mosquito Aedes aegypti. Evolution, 56, 1074-9.

Koella J.C., Rieu L. \& Paul R.E.L. (2002). Stage-specific manipulation of a mosquito's hostseeking behavior by the malaria parasite Plasmodium gallinaceum. Behav Ecol, 13, 816-820.

Koella J.C., Sorensen F.L. \& Anderson R.A. (1998). The malaria parasite, Plasmodium falciparum, increases the frequency of multiple feeding of its mosquito vector, Anopheles gambiae. Proc R Soc Lond B Biol Sci, 265, 763-8.

Koella J.C. \& Zaghloul L. (2008). Using evolutionary costs to enhance the efficacy of malaria control via genetically manipulated mosquitoes. Parasitology, 135, 1489-96.

Lacroix R., Mukabana W.R., Gouagna L.C. \& Koella J.C. (2005). Malaria infection increases attractiveness of humans to mosquitoes. PLoS Biol, 3, e298.

Lambrechts L., Chavatte J.M., Snounou G. \& Koella J.C. (2006a). Environmental influence on the genetic basis of mosquito resistance to malaria parasites. Proc Biol Sci, 273, 1501-6.

Lambrechts L., Fellous S. \& Koella J.C. (2006b). Coevolutionary interactions between host and parasite genotypes. Trends Parasitol, 22, 12-6.

Lambrechts L., Halbert J., Durand P., Gouagna L.C. \& Koella J.C. (2005). Host genotype by parasite genotype interactions underlying the resistance of anopheline mosquitoes to Plasmodium falciparum. Malar J, 4, 3 . 
Lambrechts L., Koella J.C. \& Boëte C. (2008). Can transgenic mosquitoes afford the fitness cost? Trends Parasitol, 24, 4-7.

Lambrechts L., Morlais I., Awono-Ambene P.H., Cohuet A., Simard F., Jacques J.C., Bourgouin C. \& Koella J.C. (2007). Effect of infection by Plasmodium falciparum on the melanization immune response of Anopheles gambiae. Am J Trop Med Hyg, 76, 475-80.

Lawniczak M.K., Emrich S.J., Holloway A.K., Regier A.P., Olson M., White B., Redmond S., Fulton L., Appelbaum E., Godfrey J., Farmer C., Chinwalla A., Yang S.P., Minx P., Nelson J., Kyung K., Walenz B.P., Garcia-Hernandez E., Aguiar M., Viswanathan L.D., Rogers Y.H., Strausberg R.L., Saski C.A., Lawson D., Collins F.H., Kafatos F.C., Christophides G.K., Clifton S.W., Kirkness E.F. \& Besansky N.J. (2010). Widespread divergence between incipient Anopheles gambiae species revealed by whole genome sequences. Science, 330, 512-4.

Leonard K.J. (1994). Stability of equilibria in a gene-for-gene coevolution model of hostparasite interactions. Phytopathol, 84, 70-77.

Levine N.D. (1988). The Protozoan Phylum Apicomplexa. CRC Press, Boca Raton, Florida. Liu W., Li Y., Learn G.H., Rudicell R.S., Robertson J.D., Keele B.F., Ndjango J.B., Sanz C.M., Morgan D.B., Locatelli S., Gonder M.K., Kranzusch P.J., Walsh P.D., Delaporte E., Mpoudi-Ngole E., Georgiev A.V., Muller M.N., Shaw G.M., Peeters M., Sharp P.M., Rayner J.C. \& Hahn B.H. (2010). Origin of the human malaria parasite Plasmodium falciparum in gorillas. Nature, 467, 420-5.

Lyimo E.O. \& Koella J.C. (1992). Relationship between body size of adult Anopheles gambiae s.l. and infection with the malaria parasite Plasmodium falciparum. Parasitology, 104, 233-7. 
Lyimo I.N. \& Ferguson H.M. (2009). Ecological and evolutionary determinants of host species choice in mosquito vectors. Trends Parasitol, 25, 189-96.

Macdonald G. (1957). The Epidemiology and Control of Malaria. Oxford University Press, London.

Mackinnon M.J. \& Marsh K. (2010). The selection landscape of malaria parasites. Science, $328,866-71$.

Marrelli M.T., Li C., Rasgon J.L. \& Jacobs-Lorena M. (2007). Transgenic malaria-resistant mosquitoes have a fitness advantage when feeding on Plasmodium-infected blood. Proc Natl Acad Sci U S A, 104, 5580-3.

Marshall J.M. \& Taylor C.E. (2009). Malaria control with transgenic mosquitoes. PLoS Med, $6, \mathrm{e} 20$.

Martinsen E.S., Perkins S.L. \& Schall J.J. (2008). A three-genome phylogeny of malaria parasites (Plasmodium and closely related genera): evolution of life-history traits and host switches. Mol Phylogenet Evol, 47, 261-73.

Meister S., Agianian B., Turlure F., Relogio A., Morlais I., Kafatos F.C. \& Christophides G.K. (2009). Anopheles gambiae PGRPLC-mediated defense against bacteria modulates infections with malaria parasites. PLoS Pathog, 5, e1000542.

Menge D.M., Zhong D., Guda T., Gouagna L., Githure J., Beier J. \& Yan G. (2006). Quantitative trait loci controlling refractoriness to Plasmodium falciparum in natural Anopheles gambiae mosquitoes from a malaria-endemic region in western Kenya. Genetics, 173, 235-41.

Michel K., Suwanchaichinda C., Morlais I., Lambrechts L., Cohuet A., Awono-Ambene P.H., Simard F., Fontenille D., Kanost M.R. \& Kafatos F.C. (2006). Increased melanizing activity in Anopheles gambiae does not affect development of Plasmodium falciparum. Proc Natl Acad Sci U S A, 103, 16858-63. 
Niaré O., Markianos K., Volz J., Oduol F., Toure A., Bagayoko M., Sangare D., Traore S.F., Wang R., Blass C., Dolo G., Bouare M., Kafatos F.C., Kruglyak L., Toure Y.T. \& Vernick K.D. (2002). Genetic loci affecting resistance to human malaria parasites in a West African mosquito vector population. Science, 298, 213-6.

Nirmala X. \& James A.A. (2003). Engineering Plasmodium-refractory phenotypes in mosquitoes. Trends Parasitol, 19, 384-7.

Obbard D.J., Callister D.M., Jiggins F.M., Soares D.C., Yan G. \& Little T.J. (2008). The evolution of TEP1, an exceptionally polymorphic immunity gene in Anopheles gambiae. BMC Evol Biol, 8, 274.

Paaijmans K.P., Blanford S., Bell A.S., Blanford J.I., Read A.F. \& Thomas M.B. (2010). Influence of climate on malaria transmission depends on daily temperature variation. Proc Natl Acad Sci U S A, 107, 15135-9.

Parmakelis A., Slotman M.A., Marshall J.C., Awono-Ambene P.H., Antonio-Nkondjio C., Simard F., Caccone A. \& Powell J.R. (2008). The molecular evolution of four antimalarial immune genes in the Anopheles gambiae species complex. BMC Evol Biol, 8, 79.

Prugnolle F., Durand P., Jacob K., Razakandrainibe F., Arnathau C., Villarreal D., Rousset F., de Meeus T. \& Renaud F. (2008). A comparison of Anopheles gambiae and Plasmodium falciparum genetic structure over space and time. Microbes Infect, 10, 269-75.

Prugnolle F., Durand P., Ollomo B., Duval L., Ariey F., Arnathau C., Gonzalez J.P., Leroy E. \& Renaud F. (2011). A Fresh Look at the Origin of Plasmodium falciparum, the Most Malignant Malaria Agent. PLoS Pathog, 7, e1001283. 
Rich S.M., Licht M.C., Hudson R.R. \& Ayala F.J. (1998). Malaria's Eve: evidence of a recent population bottleneck throughout the world populations of Plasmodium falciparum. Proc Natl Acad Sci U S A, 95, 4425-30.

Riehle M.M., Guelbeogo W.M., Gneme A., Eiglmeier K., Holm I., Bischoff E., Garnier T., Snyder G.M., Li X., Markianos K., Sagnon N. \& Vernick K.D. (2011). A cryptic subgroup of Anopheles gambiae is highly susceptible to human malaria parasites. Science, 331, 596-8.

Riehle M.M., Markianos K., Niare O., Xu J., Li J., Toure A.M., Podiougou B., Oduol F., Diawara S., Diallo M., Coulibaly B., Ouatara A., Kruglyak L., Traore S.F. \& Vernick K.D. (2006). Natural malaria infection in Anopheles gambiae is regulated by a single genomic control region. Science, 312, 577-9.

Rossignol P.A., Ribeiro J.M. \& Spielman A. (1984). Increased intradermal probing time in sporozoite-infected mosquitoes. Am J Trop Med Hyg, 33, 17-20.

Schmid-Hempel P. (2005). Evolutionary ecology of insect immune defenses. Annu Rev Entomol, 50, 529-51.

Sinkins S.P. \& Gould F. (2006). Gene drive systems for insect disease vectors. Nat Rev Genet, 7, 427-35.

Slotman M.A., Parmakelis A., Marshall J.C., Awono-Ambene P.H., Antonio-Nkondjo C., Simard F., Caccone A. \& Powell J.R. (2007). Patterns of selection in anti-malarial immune genes in malaria vectors: evidence for adaptive evolution in LRIM1 in Anopheles arabiensis. PLoS One, 2, e793.

Smith D.L., McKenzie F.E., Snow R.W. \& Hay S.I. (2007). Revisiting the basic reproductive number for malaria and its implications for malaria control. PLoS Biol, 5, e42.

Tanabe K., Mita T., Jombart T., Eriksson A., Horibe S., Palacpac N., Ranford-Cartwright L., Sawai H., Sakihama N., Ohmae H., Nakamura M., Ferreira M.U., Escalante A.A., 
Prugnolle F., Bjorkman A., Farnert A., Kaneko A., Horii T., Manica A., Kishino H. \& Balloux F. (2010). Plasmodium falciparum accompanied the human expansion out of Africa. Curr Biol, 20, 1283-9.

Tripet F. (2009). Ecological immunology of mosquito-malaria interactions: Of non-natural versus natural model systems and their inferences. Parasitology, 136, 1935-42.

Tripet F., Aboagye-Antwi F. \& Hurd H. (2008). Ecological immunology of mosquito-malaria interactions. Trends Parasitol, 24, 219-27.

Vernick K.D., Fujioka H., Seeley D.C., Tandler B., Aikawa M. \& Miller L.H. (1995). Plasmodium gallinaceum: a refractory mechanism of ookinete killing in the mosquito, Anopheles gambiae. Exp Parasitol, 80, 583-95.

Vittor A.Y., Pan W., Gilman R.H., Tielsch J., Glass G., Shields T., Sanchez-Lozano W., Pinedo V.V., Salas-Cobos E., Flores S. \& Patz J.A. (2009). Linking deforestation to malaria in the Amazon: characterization of the breeding habitat of the principal malaria vector, Anopheles darlingi. Am J Trop Med Hyg, 81, 5-12.

Vlachou D., Schlegelmilch T., Christophides G.K. \& Kafatos F.C. (2005). Functional genomic analysis of midgut epithelial responses in Anopheles during Plasmodium invasion. Curr Biol, 15, 1185-95.

Volkman S.K., Barry A.E., Lyons E.J., Nielsen K.M., Thomas S.M., Choi M., Thakore S.S., Day K.P., Wirth D.F. \& Hartl D.L. (2001). Recent origin of Plasmodium falciparum from a single progenitor. Science, 293, 482-4.

White B.J., Lawniczak M.K., Cheng C., Coulibaly M.B., Wilson M.D., Sagnon N., Costantini C., Simard F., Christophides G.K. \& Besansky N.J. (2010). Adaptive divergence between incipient species of Anopheles gambiae increases resistance to Plasmodium. Proc Natl Acad Sci US A, 108, 244-9. 
Windbichler N., Menichelli M., Papathanos P.A., Thyme S.B., Li H., Ulge U.Y., Hovde B.T., Baker D., Monnat R.J., Jr., Burt A. \& Crisanti A. (2011). A synthetic homing endonuclease-based gene drive system in the human malaria mosquito. Nature, 473, 212-5.

Wise de Valdez M.R., Nimmo D., Betz J., Gong H.F., James A.A., Alphey L. \& Black W.C. (2011). Genetic elimination of dengue vector mosquitoes. Proc Natl Acad Sci U S A, $108,4772-5$.

Yakob L., Bonsall M.B. \& Yan G. (2010). Modelling knowlesi malaria transmission in humans: vector preference and host competence. Malar J, 9, 329.

Yassine H. \& Osta M.A. (2010). Anopheles gambiae innate immunity. Cell Microbiol, 12, 19. 